\title{
Outcome of Endoscopic third Ventriculostomy for Obstructive Hydrocephalus due to Posterior Fossa Lesions
}

\author{
Suresh Sapkota $^{1}$, Shikher Shrestha ${ }^{1}$, Suresh Bishokarma ${ }^{1}$, Suraj Thulung ${ }^{1}$,Bibhusan Kalu ${ }^{1}$, Dinesh \\ Nath Gongal ${ }^{1}$ \\ ${ }^{1}$ Upendra Devkota Memorial Institute of Neurological and Allied Sciences, Bansbari, Kathmandu
}

Correspondence:

Dr Suresh Sapkota

Upendra Devkota Memorial Institute of Neurological and Allied Sciences, Bansbari, Kathmandu.

E-mail: Sapkota.suressh@gmail.com

Phone no: +9779841311813

\begin{abstract}
Background and purpose: Obstructive hydrocephalus can be treated with External ventricular Drainage (ETV), Ventriculo peritoneal shunt insertion, upfront tumor removal without cerebrospinal fluid diversion procedures or more recently ETV. Aim of our study was to study the outcome of ETV in treating such patients. Material and method: Descriptive observational study conducted at Upendra Devkota Memorial Institute Of Neurological and Allied Sciences from Sep 2015 to may 2017. Result: Twenty-three patients met the inclusion criteria. Among which, 14 (39\%) patients were male with male to female ratio of 1.55. We included wide age range from 3 years to 68 years. Seven patients $(30.4 \%)$ were less than 10 years while 6 patients $(23 \%)$ were above 60 years old. Patients with Posterior fossa presented with varied symptoms. Ten patients $(43.4 \%)$ presented with decreased level of consciousness, $14(60.8 \%)$ presented with visual symptoms, 17 (73.9\%) presented with vomiting and $23(100 \%)$ presented with headache (Figure. 3). Visual acuity was normal among $3(13 \%)$, decreased among $11(48 \%)$ and with no perception of light among 3 (13\%). However, acuity was not assessed among 6 (26\%) patients (Figure 4).Funduscopic evaluation revealed papilledema among $20(87 \%)$ patients while secondary optic atrophy among $3(13 \%)$ patients. Patients were evaluated in follow up for symptomatic improvement. Consciousness were improved among 8 (80\%) patients, visual symptoms improved among 10 (71.4\%) patients, vomiting subsided among $15(88.2 \%)$ patients while headache improved among 100\%. Following ETV, 5 (21.7\%) patients developed complications, $3(13 \%)$ patients had intraventricular bleeding while $2(8.6 \%)$ patients had CSF leakage from the wound. Conclusion: Endoscopic third ventriculostomy is a relatively safe and valid option for treatment obstructive hydrocephalus due to posterior fossa lesions.
\end{abstract}

Key words: ETV, Endoscopic third ventriculostomy, Posterior Fossa Tumor.

$\mathrm{E}$ ndoscopic third ventriculostomy (ETV) is a safe and minimally invasive procedure that has been used since 1993 in the treatment of many types of hydrocephalus. ${ }^{1}$ ETV has become the preferred method to treat obstructive hydrocephalus because of its minimally invasive nature. ${ }^{2}$ The most common indications include primary aqueductal stenosis ${ }^{3}$ and triventricular hydrocephalus ${ }^{2}$ resulting from external aqueductal compression and tetraventricular obstructive hydrocephalus. ${ }^{4}$

The management of hydrocephalus associated with a posterior fossa tumor is debated. ${ }^{5}$ Some authors emphasize the advantages of an immediate tumor removal that may normalize the cerebrospinal fluid while some emphasize the benefit of pre resection ETV. ${ }^{6,7}$

\section{3}

Date submitted: 05/ 11/2019

Date accepted: 18/12/2019
The purpose of the present study was to assess the surgical outcome and advantages of pre-resection ETV in permanently relieving hydrocephalus with posterior fossa tumors.

\section{Material and Methods}

Descriptive cross sectional study was designed and studied at Upendra Devkota Memorial Institute of Neurological and Allied Sciences, Bansbari, Kathmandu for 3 years from Sept 2015 to May 2017.23 patients with posterior fossa tumor with hydrocephalus who underwent ETV were included in the study. Minimal sample size of 16 was estimated using the following formula.

$\mathrm{n}=\mathrm{z} 2 \cdot \mathrm{p}(1-\mathrm{p}) / \mathrm{e} 2$, where

$\mathrm{n}=$ estimated sample size,

$\mathrm{Z}=$ confidence interval (1.65)

egneuro Volume 02, Issue 01, 2020

DOI : https://doi.org10.3126/egn.v2i1.27453 


\section{Sapkota $S$ et al.}

$\mathrm{p}=$ prevalence $=0.4$

$\mathrm{e}=$ margin of error $=0.2$

Simple random sampling technique was used. Patients Age $>6$ months with posterior fossa lesion and associated obstructive hydrocephalus were included in the study. Patients of age $\leq 6$ months, patients unfit for general anesthesia, patients with non-obstructive or communicating hydrocephalus, diagnosed/suspected hydrocephalus secondary to tubercular meningitis were excluded from the study.

\section{Dependent variables:}

Post ETV clinical improvement

Post ETV complications

Independent variables: Age, Gender

Data collection and Analysis:

All patients who underwent ETV for Hydrocephalus secondary to posterior fossa tumor were enrolled in the study. Patient demography, Post ETV clinical improvement and incidence of complications of ETV were collected from patient's hospital record. Performa was used for data entries. Analysis was done using SPSS 20. Frequencies, percentages were calculated for demographic data. Chi square/ Fisher exact test as per needed was used to evaluated the significance of association with the outcome variables.

\section{Results}

Twenty-three patients met the inclusion criteria. Among which, 14 (39\%) patients were male with male to female ration of 1.55 (Figure. 1). We included wide age range from 3 years to 68 years. Seven patients $(30.4 \%)$ were less than 10 years while 6 patients (23\%) were above 60 years old (Figure 2). Patient with Posterior fossa lesions presented with varied symptoms. Ten patients $(43.4 \%)$ presented with decreased level of consciousness, $14(60.8 \%)$ presented with visual symptoms, 17 (73.9\%) presented with vomiting and $23(100 \%)$ presented with headache (Figure. 3 ). Visual acuity was normal among 3 (13\%), decreased among $11(48 \%)$ and with no perception of light among 3 (13\%). However, acuity was not assessed among $6(26 \%)$ patients (Figure 4).Funduscopic evaluation revealed papilledema among $20(87 \%)$ patients while secondary optic atrophy among 3 (13\%) patients (Figure. 5).

Patients were evaluated in follow up for symptomatic improvement. Consciousness improved among $8(80 \%)$ patients, visual symptoms improved among $10(71.4 \%)$ patients, vomiting subsided among $15(88.2 \%)$ patients while headache improved among 100\% (Figure. 6).

Following ETV, $5(21.7 \%)$ patients developed complications, 3 (13\%) patients had intraventricular bleeding while $2(8.6 \%)$ patients had CSF leakage from the wound (Figure. 7).

\section{Discussion}

The management of hydrocephalus associated with a posterior fossa tumor is debated. ${ }^{5}$ Some authors emphasize the advantages of an immediate tumor removal that may normalize the cerebrospinal fluid (CSF) dynamics. However, in clinical practice, the mere excision of the lesion has been demonstrated to be accompanied by a persisting hydrocephalus in about one third of the cases. Internal CSF diversion using endoscopic third ventriculostomy (ETV) evolved as an appealing management option of obstructive hydrocephalus associated with posterior fossa tumors and now ETV is the procedure ofchoice for obstructive hydrocephalus ${ }^{8,9}$

ETV has numerous potential benefits over the standard shunting procedure, which possesses its own set of inherent risks and complications. Preoperative endoscopic third ventriculostomy (ETV) offers several advantages. It controls the intracranial pressure (ICP), avoids the necessity of an emergency procedure, allows appropriate scheduling of the operation for tumor removal, and eliminates the risks related to the presence of an external ventricular drainage or a permanent ventriculoperitoneal shunt. The procedure also reduces the incidence of postoperative hydrocephalus. A final advantage, more difficult to weight, but obvious to the neurosurgeon, is the possibility of removing the lesion with a relaxed brain and normal ICP.

Patient with posterior fossa presented with varied symptoms. In our study, 10 patients (43.4\%) presented with decreased level of consciousness, 
$14(60.8 \%)$ presented with visual symptoms, 17 (73.9\%) presented with vomiting and 23 (100\%) presented with headache.

Visual acuity was normal among 3 (13\%), decreased among $11(48 \%)$ and with no perception of light among 3 (13\%). However, acuity was not assessed among $6(26 \%)$ patients.

Funduscopic evaluation revealed papilledema among 20 (87\%) patients while secondary optic atrophy among $3(13 \%)$ patients.

\section{Improvement}

Patients were evaluated in follow up at 7days for symptomatic improvement. Consciousness was improved among 8 (80\%) patients; visual symptoms improved among 10 (71.4\%) patients, vomiting subsided among $15(88.2 \%)$ patients while headache improved among $100 \%$ of them.

Following ETV, 5 (21.7\%) patients developed complications, 3 (13\%) patients had intraventricular bleeding while $2(8.6 \%)$ patients had CSF leakage from the wound. In a study done by Buxton et al., among 63 patients, they experienced complication among 7 (11\%) patients including ventriculitis (1.6\%), cerebral infarction (1.6\%), intraoperative haemorrhage requiring EVD $(1.6 \%)$, bilateral chronic subdural haematomas $(1.6 \%)$ and death $(4.7 \%) .{ }^{10}$ In our study, there was no mortality.

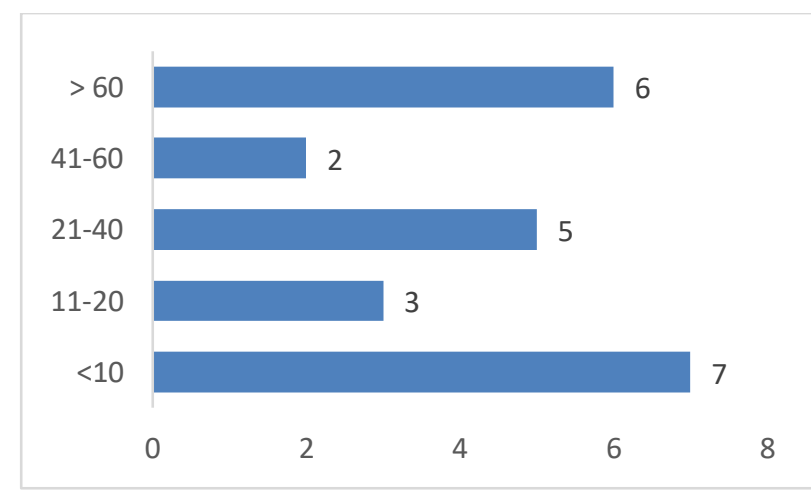

Figure 1. Age distribution in years among study populations $(n=23)$

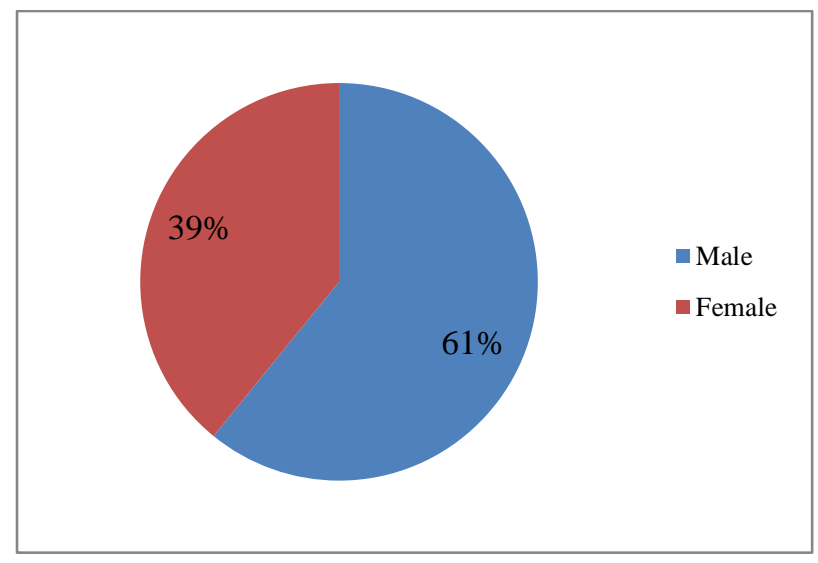

Figure 2. Sex distribution $(\mathrm{N}=23)$

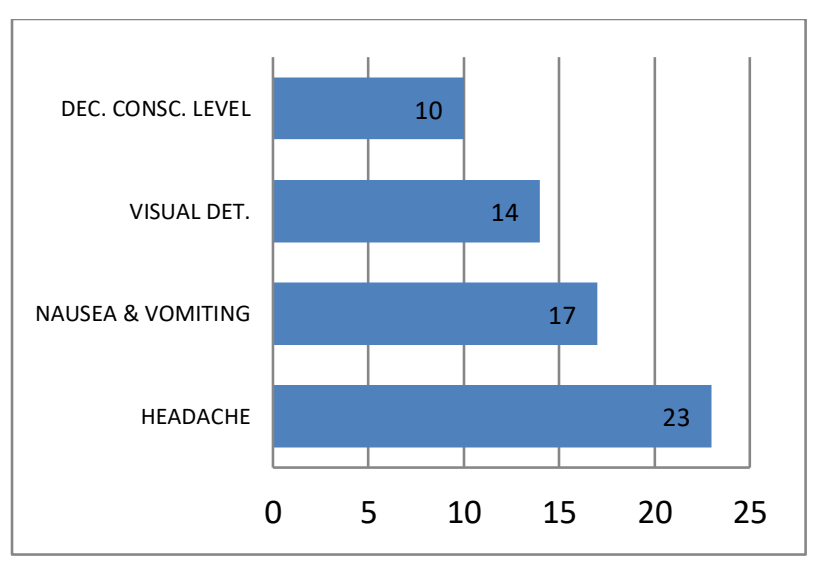

Figure 3. Symptomatology of patients $(n=23)$ Dec. Consc. Level-Decreased conscious level Visual Det-Visual deterioration

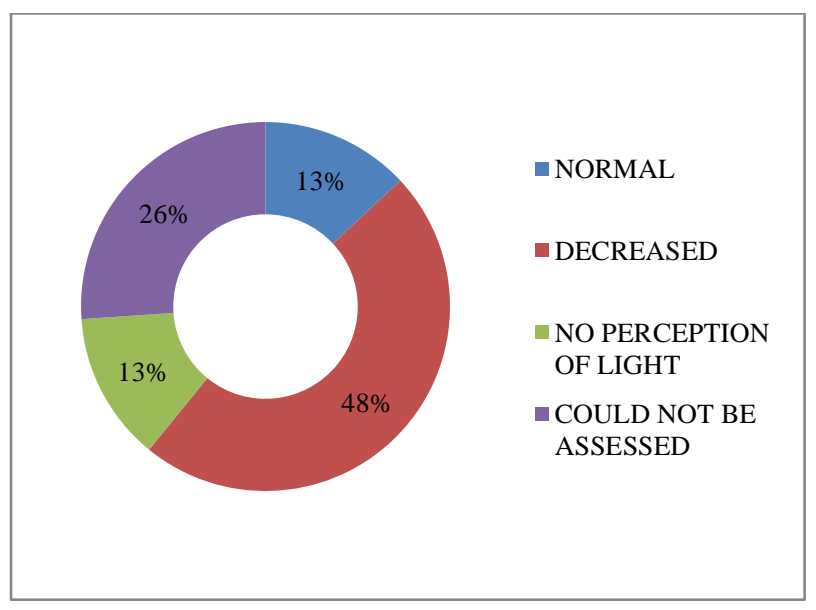

Figure 4. Visual acuity of patients $(n=23)$ 


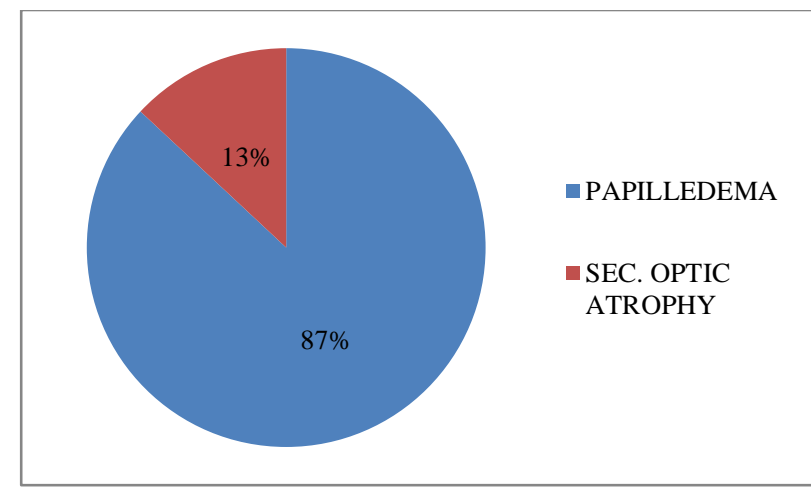

Figure 5. Funduscopic evaluation of patient with hydrocephalus $(n=23)$

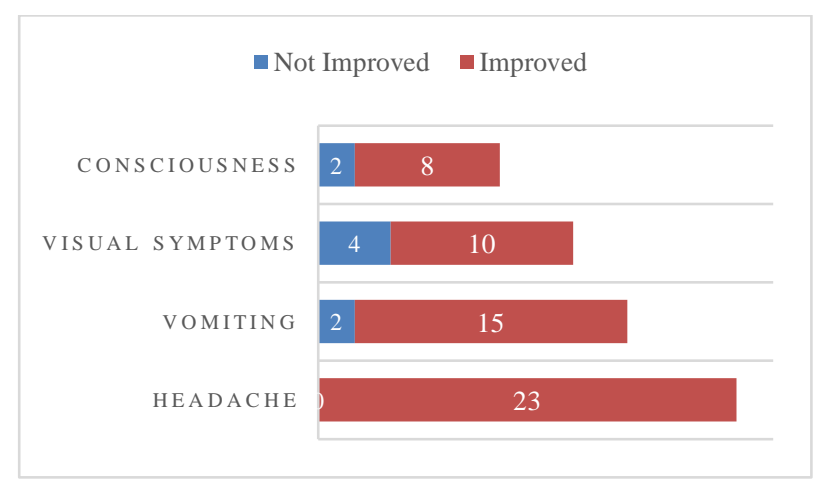

Figure 6. Clinical improvement following ETV $(n=23)$

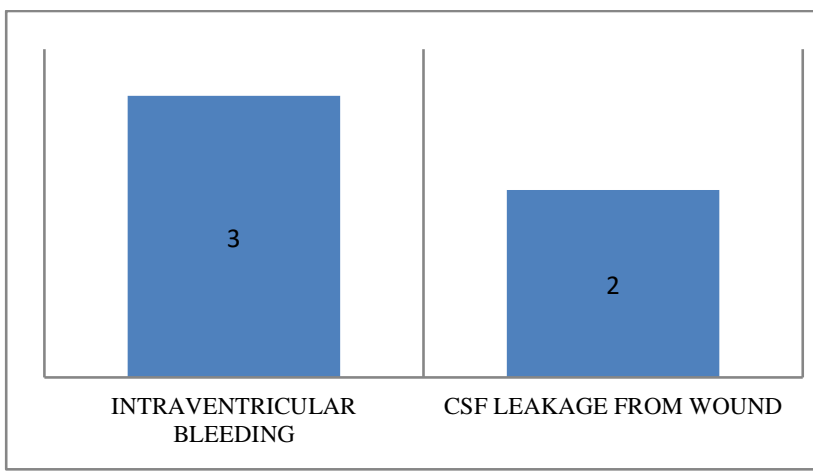

Figure 7. Chart showing post ETV complications $(\mathrm{n}=23)$

\section{Limitations:}

This is a restrospective observational study based on a single institution review. Practices for the treatment of obstructive hydrocephalus are varied and other possibilities have not been reviewed. A larger study with consideration of other treatment options like VP shunt or an external ventricular drainage should be considered and compared with each other for optimal results.

\section{Conclusion}

Endoscopic third ventriculostomy is a relatively safe and valid option for treatment obstructive hydrocephalus due to posterior fossa lesions.

\section{References}

1. Balevi M. Endoscopic Third Ventriculostomy in Normal Pressure Hydrocephalus and Symptomatic Long-standing Overt Ventriculomegaly. Asian J Neurosurg. 2017;12(4):605-12.

2. Wagshul ME, McAllister JP, Rashid S, Li J, Egnor MR, Walker ML, et al. Ventricular dilation and elevated aqueductal pulsations in a new experimental model of communicating hydrocephalus. Exp Neurol. 2009;218(1):33-40.

3. da Silva LR, Cavalheiro S, Zymberg ST. Endoscopic aqueductoplasty in the treatment of aqueductal stenosis. Childs Nerv Syst 2007;23:1263-8.

4. Gangemi M, Maiuri F, Buonamassa S, Colella G, de Divitiis E. Endoscopic third ventriculostomy in idiopathic normal pressure hydrocephalus. Neurosurgery. 2004;55(1):129-34; discussion 34.

5. Di Rocco F, Juca CE, Zerah M, Sainte-Rose C. Endoscopic third ventriculostomy and posterior fossa tumors. World Neurosurg. 2013;79(2 Suppl):S18 e5-9.

6. Ruggiero C, Cinalli G, Spennato P, Aliberti F, Cianciulli E, Trischitta V, et al. Endoscopic third ventriculostomy in the treatment of hydrocephalus in posterior fossa tumors in children. Childs Nerv Syst. 2004;20(11-12):828-33.

7. Azab W, Al-Sheikh T, Yahia A. Preoperative endoscopic third ventriculostomy in children with posterior fossa tumors: an institution experience. Turk Neurosurg. 2013;23(3):359-65.

8. Sainte-Rose C, Cinalli G, Roux FE, Maixner R, Chumas PD, Mansour $\mathrm{M}$, et al. Management of hydrocephalus in pediatric patients with posterior fossa tumors: the role of endoscopic third ventriculostomy. J Neurosurg. 2001;95(5):791-7.

9. Sacko O, Boetto S, Lauwers-Cances V, Dupuy M, Roux FE. Endoscopic third ventriculostomy: outcome analysis in 368 procedures. J Neurosurg Pediatr. 2010;5(1):68-74.

10. Buxton N, Ho KJ, Macarthur D, Vloeberghs M, Punt J, Robertson I. Neuroendoscopic third ventriculostomy for hydrocephalus in adults: report of a single unit's experience with 63 cases. Surg Neurol. 2001;55(2):74-8.

egneuro, Volume 02, Issue 01, 2020 